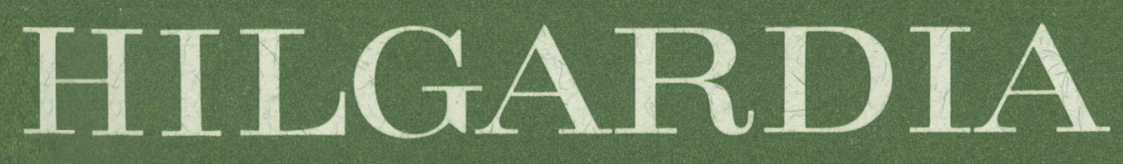

A JOURNAL OF AGRICULTURAL SCIENCE PUBLISHED BY THE CALIFORNIA AGRICULTURAL EXPERIMENT STATION

Volume $52 \cdot$ Number 3 - February 1984

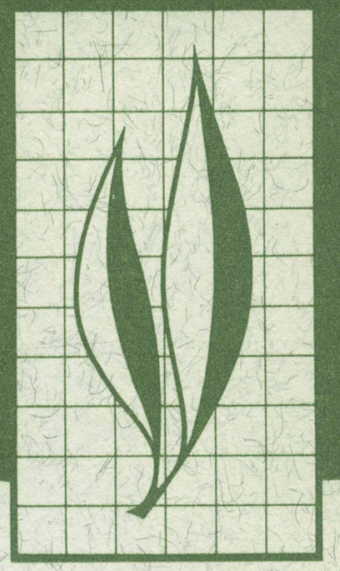

\title{
Ecology of Gall-forming Lepidoptera
} on Tetradymia

I. Gall Size and Shape II. Plant Stress Effects on Infestation Intensity

III. Within-plant Horizontal and Vertical Distribution

Hollister Hartman 


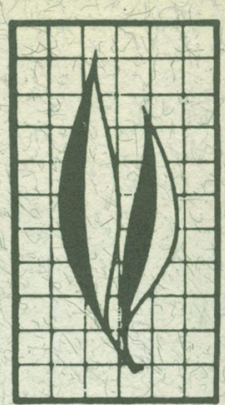

\section{Gall Size and Shape}

Multiway analysis of covariance revealed ecological determinants of gall morphology operating hierarchically, with plant effects primary, insect effects secondary, and habitat effects tertiary. Plant effects included Tetradymia DC. (Asteraceae) species identity, timing and duration of growth pulses, and differential vegetation quality among height strata. Insect effects included a numerical response in gall density and a functional response in individual feeding duration and intensity. Alterations in Gnorimoschema ketradymiella Busck 1903 (Lepidoptera: Gelechiidae) feeding caused by parasitization created parasitoid-specific gall sizes. Habitat effects indexed by topographic and climatic features operated most strongly in relatively southern latitudes.

\section{Plant Stress Effects on Infestation Intensity}

Hypothesized differential genotypic galling susceptibility of Tetradymia stenolepis Greene 1885 was not confirmed by tests of correlations between sample size and sample variance in plant-mean densities of Scrobipalpa n. sp. leaf galls and Gnorimoschema tetradymiella Busck 1903 stem galls. An alternate conceptual model, the stress-heterogeneity hypothesis, received support. The greater the stress experienced by a plant subpopulation, the more discontinuous was the frequency distribution of its plant-mean gall abundance. Also according to the model, gall formation increased with stress until a physiological threshold was exceeded, after which it dropped precipitously. Covariance analysis enabled comparisons among plots. Encounter frequency and resource density were also implicated in predicting plant-mean and branch-mean gall densities.

\section{THE AUTHOR:}

Hollister Hartman, formerly research assistant with the Population Biology Program, Department of Entomology, University of California, Riverside, is with TRW, Ballistic Missiles Division, P.O. Box 1310, San Bernardino, CA 92402. 


\section{Ecology of Gall-forming Lepidoptera on Tetradymia ${ }^{1,2}$}

\section{Gall Size and Shape}

\section{INTRODUCTION}

Plant galls RePresent a unique interspecific interaction between a plant species and a gall-inducing organism. Cosens (1913) defined galls (also known as cecidia) as "any enlargement of plant cells, tissues, or organs induced by the stimulus of a parasitic organism as a regular incident in the life history of the parasite." Galls arise due to hypertrophy (overgrowth) and hyperplasy (cell proliferation) of the tissue affected.

Two kinds of galls were investigated: Stem galls formed by the gelechiid moth Gnorimoschema tetradymiella Busck 1903 and galls formed from individual leaves by the gelechiid moth Scrobipalpa n. sp. Overwintering eggs of G. tetradymiella hatch during April, stem gall formation and larval growth continue through June, pupation takes place in the gall during June and July, and the gray-mottled, 8-10 mm adults are active, mainly during early-morning hours, until mid-August. Second generation larvae feed from mid-June to mid-September, followed by pupation between mid-August and mid-October. Adults emerge again starting in mid-September and oviposition continues until the onset of cold weather, usually November. Further details are given in Hartman (1982a).

Larval feeding and leaf gall formation by Scrobipalpa n. sp. commence in mid-March. In mid-April the larvae begin chewing out of their galls and constructing sand cells in which to pupate about $1 \mathrm{~cm}$ under the surface of the soil. By mid-May most of the population is underground. Adults, brown and 5-6 mm long, emerge 5 to 6 weeks later, ready to mate. In the absence of a suitable host plant, oviposition does not occur. In the related species, S. ocellatella Boyd, mean fecundity on sugar beet is 134 eggs/female; host plant chemical signals also determine oviposition site and behavior (Robert and Blaisinger, 1978).

The shrub Tetradymia DC. (Asteraceae) is found only in the western half of the United States. According to the recent revision by Strother (1974), there are ten species, six of which occur in southern California. I studied T. stenolepis Greene 1885, T. axillaris Nels. var. longispina (Jones 1895) Strother and T. glabrata Torr. \& Gray 1855 because all three are desert specialists, often sympatric, and gall-infested by the same guild of gelechiid moths. Tetradymia axillaris is slightly larger (to $1.5 \mathrm{~m}$ high) than both $T$. stenolepis and glabrata (to $1.2 \mathrm{~m}$ high). Tetradymia stenolepis ranges the lowest in elevation (to $600 \mathrm{~m}$ ), and glabrata, the highest (to $2400 \mathrm{~m}$ ). Tetradymia axillaris flowers as early as April, while $T$. stenolepis flowers as late as August. Geographically, T. glabrata occurs widely both inside and outside of California, whereas T. stenolepis is found solely within southern California (Strother, 1974). Galls occurred virtually wherever Tetradymia was found (Hartman, 1982a, Appendix 1).

\footnotetext{
${ }^{1}$ This paper is part of a thesis submitted in partial fulfillment of the Ph.D. degree, University of California, Riverside, December 1982.

${ }^{2}$ Accepted for publication July 18, 1983
} 
Galls of Lepidoptera generally are fusiform hollow swellings which persist long after the escape of the adult. Eggs are deposited on the surface of a tender plant part, and galls usually arise near or at the growing tip, surmounted by tufts of stunted branch rudiments (Mani, 1964). Stem galls on Tetradymia were oblate to fusiform, covered by dense woolly pubescense; leaf galls were hollow sessile green spheres when new, becoming wrinkled and brown after drying. The physical features of a collection of galls revealed information about their ecosystem of origin, intensity and type of parasitization, long- and short-term weather changes, and soil moisture conditions.

My main objective was to determine how stem gall size and shape depended on Tetradymia species identity and locality, gall inhabitants, and time of year. In conjunction with this goal I distinguished morphological traits causally related to the above variables from those simply "carried along" by virtue of allometric constraints. A secondary objective was to quantify the influence of gall height and number on leaf and stem gall length.

\section{METHODS}

A stratified random sample of ca. 170 stem galls was collected from each of five localities (Table I.1, Fig. I.1) in September 1980. 1) Maximum length and diameter were recorded to the nearest $\mathrm{mm}$; 2) sprouts from the gall's surface were removed, and their number and mean length to the nearest $\mathrm{mm}$ recorded; 3) spines were removed, and their number and mean length to the nearest $\mathrm{mm}$ recorded; 4) the gall was split lengthwise with clippers and opened carefully so as not to disturb the gall contents; 5) chamber length was recorded to the nearest $\mathrm{mm}$, and chamber diameter to the nearest $0.5 \mathrm{~mm}$; 6) gall contents were inspected under a dissecting microscope and their identities or a detailed description recorded.

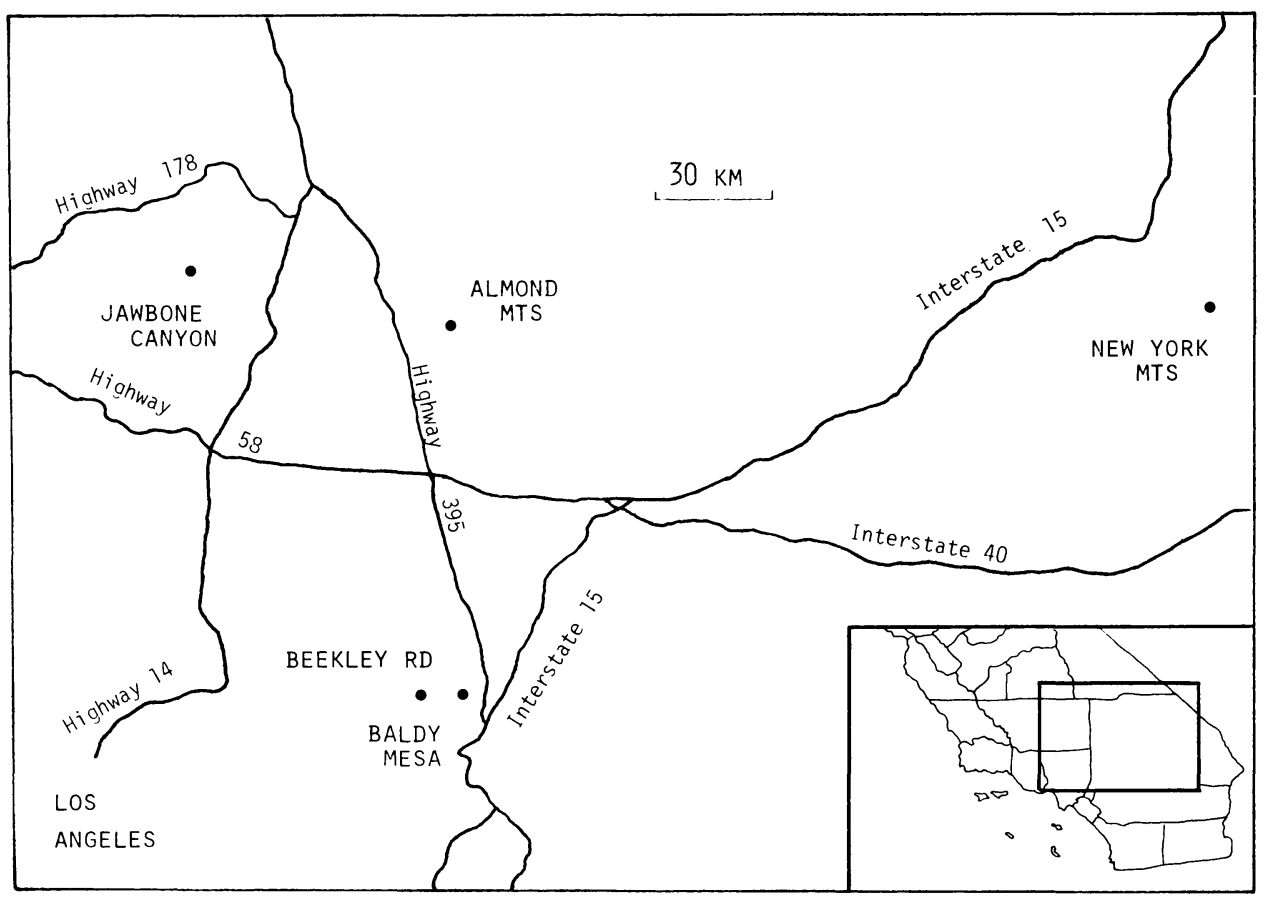

Fig. I.1. Location of main study sites in southern California. 
TABLE I.1. DESCRIPTION OF STUDY AREAS IN SOUTHERN CALIFORNIA

\begin{tabular}{lcccccc}
\hline \hline \multicolumn{7}{c}{ Topography } \\
\hline Site* & $\begin{array}{c}\text { Elevation } \\
(\mathrm{m})\end{array}$ & $\begin{array}{c}\text { Slope } \\
\text { facing }\end{array}$ & $\begin{array}{c}\text { Degree } \\
\text { slope } \\
(\mathrm{m} / 30 \mathrm{~m})\end{array}$ & $\begin{array}{c}\text { Latitude } \\
(\text { relative } \\
\left.\text { to } 35^{\circ} \mathrm{N}\right)\end{array}$ & $\begin{array}{c}\text { Longitude } \\
\text { (W of New } \\
\text { York Mts.) }\end{array}$ & $\begin{array}{c}\text { Mean annual } \\
\text { temperature } \\
\left(\text { wrt } 14^{\circ} \mathrm{C}\right)\end{array}$ \\
\hline AM & 800 & SW & 0.65 & $\mathrm{~N}$ & $2.13^{\circ}$ & high \\
BM & 1055 & $\mathrm{~N}$ & 0.51 & $\mathrm{~S}$ & 2.10 & high \\
BR & 1237 & $\mathrm{~N}$ & 1.11 & $\mathrm{~S}$ & 2.28 & high \\
JC1 & 1219 & SW & 0.47 & $\mathrm{~N}$ & 2.89 & low \\
JC2 & 1244 & SW & 0.62 & $\mathrm{~N}$ & 2.89 & low \\
NY1 & 1122 & $\mathrm{NW}$ & 1.33 & $\mathrm{~N}$ & 0 & low \\
NY2 & 1138 & $\mathrm{NW}$ & 1.33 & $\mathrm{~N}$ & 0 & low \\
\hline
\end{tabular}

\begin{tabular}{lccccc}
\hline \multicolumn{6}{c}{ Vegetation } \\
\hline Site* & $\begin{array}{c}\text { Density } \\
\text { (plants/30 } \\
\text { linear m) }\end{array}$ & $\begin{array}{c}\text { Height } \\
\text { mean }(\mathbf{c m})\end{array}$ & $\begin{array}{c}\text { Height } \\
\text { variance } \\
(\mathbf{s q} \mathbf{~ c m})\end{array}$ & $\begin{array}{c}\text { Diversity } \\
\mathbf{H}^{\prime}=-\left(\mathbf{p}_{\mathbf{i}} \log \mathbf{p}_{\mathbf{i}}\right)\end{array}$ & $\begin{array}{c}\text { Cover } \\
(\% \text { area })\end{array}$ \\
\hline AM & 8 & 60.8 & 387 & .566 & 13.3 \\
BM & 31 & 50.2 & 1133 & .912 & 40.0 \\
BR & 21 & 60.9 & 371 & .682 & 32.5 \\
JC1 & 25 & 51.7 & 324 & .413 & 30.0 \\
JC2 & 21 & 52.8 & 527 & .634 & 23.0 \\
NY1 & 51 & 42.3 & 1439 & .859 & 62.5 \\
NY2 & 53 & 37.8 & 1157 & .732 & 56.5 \\
\hline
\end{tabular}

\begin{tabular}{llccccc}
\hline Insecta $\dagger$ \\
Site* & $\begin{array}{c}\text { Host plant } \\
\text { species }\end{array}$ & $\begin{array}{c}\text { Mean } \\
\text { instar } \neq\end{array}$ & $\begin{array}{c}\text { Wing } \\
\text { length } \\
(\mathbf{m m})\end{array}$ & $\begin{array}{c}\text { Total \% } \\
\text { mortality }\end{array}$ & $\begin{array}{c}\text { Parasitism } \\
\text { \% of } \\
\text { mortality }\end{array}$ & $\begin{array}{c}\text { Inquiline } \\
\text { diversity } \\
\mathbf{H}^{\prime}=-\left(\mathbf{p}_{\mathbf{i}} \text { In }_{\mathbf{i}}\right)\end{array}$ \\
\hline AM & glabrata & 2.68 & 9.31 & 58.5 & 69.4 & 1.897 \\
AM & stenolepis & 2.62 & 8.19 & 48.0 & 95.8 & 1.637 \\
BM & axillaris & 2.42 & 8.57 & 47.2 & 84.0 & - \\
BM & stenolepis & 1.60 & 7.64 & 50.0 & 80.9 & 1.422 \\
BR & stenolepis & 2.18 & 7.71 & 64.8 & 70.4 & 1.713 \\
JC1 & axillaris & 2.51 & 8.65 & 69.0 & 76.9 & 1.837 \\
JC1 & stenolepis & 2.18 & 8.16 & 71.4 & 65.9 & 2.114 \\
JC2 & axillaris & 2.33 & 8.30 & 81.1 & 77.9 & 1.760 \\
NY1 & stenolepis & 2.29 & 8.17 & 72.0 & 83.3 & 1.340 \\
NY2 & stenolepis & 2.13 & 7.50 & 67.1 & 63.8 & 1.414 \\
\hline
\end{tabular}

*AM = Almond Mts, $8.8 \mathrm{~km} \mathrm{~W}$ of Blackwater well (San Bernardino County)

$\mathrm{BM}=$ Baldy Mesa Road, $2.7 \mathrm{~km}$ N of Phelan Road (San Bernardino County)

$\mathrm{BR}=$ Beekley Road, $1.6 \mathrm{~km}$ N of Phelan Road (San Bernardino County)

$\mathrm{JC} 1$ = Jawbone Canyon Road, Jct. Weldon/Piute (Kern County)

JC2 = Jawbone Canyon Road, $2.3 \mathrm{~km} \mathrm{NW}$ of JC1 (Kern County)

NY1 $=$ New York Mts, $1.6 \mathrm{~km} \mathrm{~S}$ of Ivanpah (San Bernardino County)

NY2 $=$ New York Mts, $0.4 \mathrm{~km} \mathrm{~S}$ of NY1 (San Bernardino County)

+ Gnorimoschema tetradymiella stem gallmakers on Tetradymia.

$\ddagger 1=$ early instar, 2 = late instar, 3 = pupa. 
Means of the above measures were compared among host plant species using Duncan's multiple range test. Partial correlations between each pair of dimensions were then factoranalyzed, holding constant: 1) topographic slope, which was locality-specific (and significantly related to chamber width, number of spines, spine length, number of sprouts and sprout length); and 2) gall length-to-width ratio, which was highly species-specific.

Due to analysis of variance interaction of gall content with both latitude and elevation, effects of gall inhabitants on gall morphology were examined within each locality. Statistical controls for Tetradymia species, slope, elevation, and latitude were applied to deviations in gall length and chamber width to summarize inhabitant effects.

Northern and southern topographic effects on gall morphology were also studied separately due to ANOVA interactions of latitude with the other factors. Multiple classification analysis supplied by the Statistical Package for the Social Sciences (SPSS; Nie et al., 1975) allowed assessment of slope and elevation effects, ignoring differences in plant and insect species-composition among sites.

For each gall type (new leaf, old leaf, new stem, old stem) and elevation $(1122,1138$, $1268 \mathrm{~m}$ ) sampled in the New York Mountains, California, one way ANOVA's were performed with respect to mean gall size, gall height, and relative height ( $=$ gall height/plant height) over four compass quadrants (NE, NW, SW, SE). Correlations were calculated for all possible pairs of variables, with $n=12$ for each coefficient.

\section{RESULTS}

Characters which discriminated T. axillaris, glabrata, and stenolepis stem galls included gall width, chamber length, length-to-width ratio and wall thickness (Table I.2). Spine length, sprout number, and sprout length were so variable they did not differentiate the species.

Factor analysis of raw correlations among plot-mean values for each trait yielded three independent dimensions: gall and chamber length ( $\mathrm{x}$-axis), chamber width and sprout number (y-axis), and spine length (z-axis). Factor analysis of the above-described partial correlations retained bidimensionality (Fig. I.2), demonstrating that stem gall morphology

TABLE I.2. DUNCAN'S MULTIPLE RANGE TESTS OF TETRADYMIA STEM GALL MEASURES BY HOST PLANT SPECIES

\begin{tabular}{lccc}
\hline \hline & \multicolumn{3}{c}{ Character mean } \\
\cline { 2 - 4 } Gall character & T. stenolepis & T. axillaris & T. glabrata \\
\hline Length (mm)* & 16.7 & 14.0 & 23.4 \\
Width (mm)** & 9.3 & 10.5 & 5.7 \\
Spine number* & 9.8 & 2.2 & 9.6 \\
Spine length (mm) & 15.4 & 18.2 & 12.3 \\
Sprout number & 1.8 & 1.0 & $1.0 \dagger$ \\
Sprout length $(\mathrm{cm})$ & 5.7 & 16.4 & 2.3 \\
Chamber length (mm)** & 14.0 & 11.2 & 21.4 \\
Chamber width (mm)* & 4.9 & 3.4 & 3.7 \\
Length/width** & 1.85 & 1.35 & 4.18 \\
Wall thickness** & 2.19 & 3.53 & 1.00 \\
\hline
\end{tabular}

*Significant at .05 level.

**Significant at .01 level.

†Highly variable.

$\ddagger$ Wall thickness $=($ width minus chamber width $) / 2$. 
still varied significantly beyond simple host species differences. Gall length and chamber width were selected for further analysis.

The nine most common types of stem gall inhabitant (Table I.3) included 712, or 84.0 percent of the galls. The remaining 136 cases were discarded to limit comparisons across study sites to parallel insect effects on gall morphology. (Complete data listings are given in Hartman, 1982b.)

Analysis of variance explained 50 percent of the variation in gall length and 40 percent in chamber width with respect to the five main effects: Host plant species was the most important factor for both dimensions $(61.1 \%$ and $65.3 \%$ of explained, respectively), followed by gall inhabitant ( $19.6 \%$ and $31.7 \%$ of explained, respectively). Slope explained a

Fig. I.2. Factor analysis of stem gall measures over all study sites and host plant species, controlling for site-specific and host-specific effects via partial correlation. Connections between points indicate correlations with probability less than .025 .

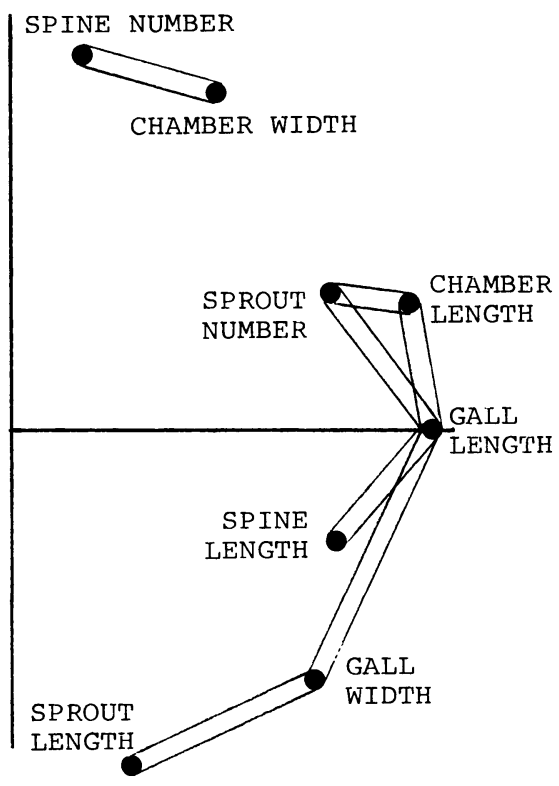

TABLE I.3. COMPARISON OF TETRADYMIA STEM GALL INHABITANTS FROM DIFFERENT HOST PLANT SPECIES POOLED FOR ALL STUDY SITES, SEPTEMBER 1980

\begin{tabular}{|c|c|c|c|c|}
\hline \multirow[b]{2}{*}{ Gall inhabitant } & \multicolumn{3}{|c|}{ Number of galls } & \multirow{2}{*}{$\begin{array}{l}\text { Percent } \\
\text { of total* }\end{array}$} \\
\hline & T. stenolepis & T. axillaris & T. glabrata & \\
\hline \multicolumn{5}{|c|}{ Gnorimoschema tetradymiella } \\
\hline Empty & 58 & 30 & 24 & 15.7 \\
\hline Early instar & 75 & 9 & 9 & 13.1 \\
\hline Late instar & 29 & 22 & 0 & 7.2 \\
\hline Pupa & 88 & 34 & 37 & 22.3 \\
\hline \multicolumn{5}{|l|}{ Parasite/predator $\dagger$} \\
\hline Copidosoma & 111 & 4 & 0 & 16.2 \\
\hline Lissonota & 18 & 83 & 0 & 14.2 \\
\hline Apanteles & 0 & 0 & 21 & 2.9 \\
\hline Unknown a & 19 & 5 & 7 & 4.4 \\
\hline \multirow[t]{2}{*}{ Unknown $b$} & 29 & 0 & 0 & 4.1 \\
\hline & 427 & 187 & 98 & \\
\hline
\end{tabular}

*Percentages may not sum to 1.0 due to rounding.

+ Copidosoma $=$ Chalcidoidea: Encyrtidae; Lissonota = Ichneumonoidea: Ichneumonidae; Apanteles $=$ Ichneumonoidea: Braconidae; Unknown = segregated by appearance. 
small but significant fraction of both variances $(3.3 \%$ and $2.8 \%$ of explained, respectively), whereas elevation was important only for gall length (16.0\% of explained). Once these factors were taken into account, latitude per se had no influence on gall morphology.

In analyzing insect effects on gall length at each locality (Table I.4), deviations (adjusted by covariance analysis) of at least 90 percent of the $1 \mathrm{~mm}$ measurement precision, or 0.9 $\mathrm{mm}$, were heuristically defined as biologically important. Likewise, deviations in chamber width (recorded to the nearest $0.5 \mathrm{~mm}$ ) of at least $0.45 \mathrm{~mm}$ were considered important. The magnitude of deviations caused by an inhabitant sometimes differed among sites, but the direction of significant values usually did not.

Insect behavior in choice of gall site also influenced gall size. In the New York Mountains, intraplant quad location of new leaf galls caused significant differences in gall size and/or height at each elevation. At $1122 \mathrm{~m}$ and $1138 \mathrm{~m}$ in 1977, relative height was lowest in southern quadrants ( $59.3 \%$ to $60.5 \%$ of plant height) and greatest in northern quadrants $(62.9 \%$ to $69.6 \%$ ) (Table I. $)$.

Quad-specific new leaf gall height and size patterns at $1268 \mathrm{~m}$ differed from those at $1122 \mathrm{~m}$ and $1138 \mathrm{~m}$. First, relative height was lowest for $\mathrm{NW}$-facing rather than southfacing galls. Second, mean relative height averaged only 51.5 percent (S.D. $=25.2 \%$ ) as

TABLE I.4. MULTIPLE CLASSIFICATION ANALYSIS OF STEM GALL MORPHOLOGY BY INHABITANT, ADJUSTED FOR HOST PLANT SPECIES WITHIN EACH STUDY SITE, SEPTEMBER 1980

\begin{tabular}{lccccc}
\hline \hline & \multicolumn{5}{c}{ Deviation from grand mean gall length (mm) } \\
\cline { 2 - 6 } Gall inhabitant & $\begin{array}{c}\text { Almond } \\
\text { Mts. }\end{array}$ & $\begin{array}{c}\text { Baldy } \\
\text { Mesa }\end{array}$ & $\begin{array}{c}\text { Beekley } \\
\text { Road }\end{array}$ & $\begin{array}{c}\text { Jawbone } \\
\text { Canyon }\end{array}$ & $\begin{array}{c}\text { New York } \\
\text { Mts. }\end{array}$ \\
\hline Gnorimoschem & tetradymiella & & & & \\
Empty & 1.03 & -0.59 & 0.32 & -1.58 & 0.38 \\
Early instar & -1.03 & -1.57 & -0.19 & -1.11 & -0.93 \\
Late instar & 0.82 & -0.12 & 0.70 & -0.90 & -0.24 \\
Pupa & 0.40 & -0.27 & -0.78 & -1.04 & -1.80 \\
Parasite/predator & & & & & \\
Copidosoma & 3.57 & 1.87 & 1.20 & 3.29 & 0.86 \\
Lissonota & 1.40 & 0.62 & $*$ & 0.55 & 2.03 \\
Apanteles & -1.97 & $*$ & $*$ & $*$ & $*$ \\
Unknown a & -2.01 & $*$ & $*$ & 0.40 & -1.39 \\
Unknown b & $*$ & $*$ & -0.23 & $*$ & $*$ \\
\hline
\end{tabular}

Deviation from grand mean chamber width $(\mathrm{mm})$

\begin{tabular}{lrrrrr}
\cline { 2 - 6 } & $\begin{array}{c}\text { Almond } \\
\text { Mts. }\end{array}$ & $\begin{array}{c}\text { Baldy } \\
\text { Mesa }\end{array}$ & $\begin{array}{c}\text { Beekley } \\
\text { Road }\end{array}$ & $\begin{array}{c}\text { Jawbone } \\
\text { Canyon }\end{array}$ & $\begin{array}{c}\text { New York } \\
\text { Mts. }\end{array}$ \\
\hline $\begin{array}{l}\text { G. tetradymiella } \\
\text { Empty }\end{array}$ & 0.11 & -0.50 & -0.90 & 0.09 & -1.04 \\
Early instar & -0.86 & 0.05 & 0.37 & -0.85 & 0.14 \\
Late instar & 0.34 & -0.57 & 0.66 & 0.07 & -0.31 \\
Pupa & 0.29 & 0.44 & 0.07 & 0.13 & 0.07 \\
Parasite/predator & & & & & \\
$\quad$ Copidosoma & 1.43 & 0.01 & 0.36 & 0.30 & 0.55 \\
Lissonota & -0.12 & 0.19 & $*$ & 0.07 & -0.18 \\
Apanteles & -0.62 & $*$ & $*$ & $*$ & $*$ \\
Unknown a & -0.01 & $*$ & $*$ & -0.12 & -0.16 \\
Unknown b & $*$ & $*$ & 0.08 & $*$ & $*$ \\
\hline
\end{tabular}

*Absent from locality. 
compared to the overall relative height of 62.7 percent (S.D. $=18.8 \%$ ) at $1122 \mathrm{~m}$ and $1138 \mathrm{~m}$. Third, unlike the trend at $1122 \mathrm{~m}$ and $1138 \mathrm{~m}$ that the lower the quad-mean relative height, the larger the galls, at $1268 \mathrm{~m}$ galls were both the smallest and the lowest of all three elevations.

A significant quad effect for old leaf galls sampled in 1977 was observed only at $1138 \mathrm{~m}$. Here, the largest galls occurred to the east, especially the SE (Table I.5). This was opposed to the new gall pattern at $1122 \mathrm{~m}$, where the largest galls occurred to the west. The unusual bias of leaf galls sampled in 1977 toward the SW quad, versus the skewness in other years toward the SE quad, is a possible explanation for the above anomaly (see also Hartman, 1983c).

TABLE I.5. ELEVATION-MEAN GALL SIZE, HEIGHT, AND RELATIVE HEIGHT PER QUAD FOR T. STENOLEPIS IN THE NEW YORK MOUNTAINS, 1977 TO 1978

\begin{tabular}{|c|c|c|c|c|c|c|}
\hline \multirow[b]{2}{*}{ Year } & \multirow[b]{2}{*}{ Elevation } & \multirow[b]{2}{*}{ Quad } & \multicolumn{4}{|c|}{$\begin{array}{c}\text { New leaf gall } \\
\text { Quad-means, associated F tests }\end{array}$} \\
\hline & & & Size & Height & Rel. ht. & Number \\
\hline \multirow[t]{15}{*}{1977} & \multirow[t]{5}{*}{$1122 \mathrm{~m}$} & $\mathrm{NE}$ & $5.66 \mathrm{~mm}$ & $38.79 \mathrm{~cm}$ & $65.53 \%$ & 96 \\
\hline & & NW & 6.17 & 34.85 & 62.87 & 72 \\
\hline & & SW & 6.04 & 34.06 & 59.53 & 72 \\
\hline & & SE & 5.58 & 36.02 & 59.28 & 77 \\
\hline & & $\mathrm{F}=$ & $\begin{array}{c}3.33 \\
(\mathrm{p}=.020)\end{array}$ & $\begin{array}{c}2.625 \\
(\mathrm{p}=.051)\end{array}$ & $\begin{array}{c}2.268 \\
(\mathrm{p}=.081)\end{array}$ & 317 \\
\hline & \multirow[t]{5}{*}{1138} & $\mathrm{NE}$ & 5.72 & 31.39 & 69.56 & 29 \\
\hline & & NW & 6.00 & 31.00 & 67.74 & 38 \\
\hline & & SW & 6.29 & 27.58 & 60.47 & 58 \\
\hline & & $\mathrm{SE}$ & 6.25 & 27.25 & 59.63 & 56 \\
\hline & & $\mathrm{F}=$ & $\begin{array}{c}0.958 \\
(\mathrm{p}=.414)\end{array}$ & $\begin{array}{c}1.690 \\
(p=.171)\end{array}$ & $\begin{array}{c}2.794 \\
(p=.042)\end{array}$ & 181 \\
\hline & \multirow[t]{5}{*}{1268} & $\mathrm{NE}$ & 5.30 & 27.94 & 58.20 & 23 \\
\hline & & NW & 5.33 & 20.32 & 41.14 & 21 \\
\hline & & SW & 5.39 & 25.10 & 54.05 & 33 \\
\hline & & SE & 4.77 & 27.15 & 50.68 & 26 \\
\hline & & $\mathrm{F}=$ & $\begin{array}{c}1.751 \\
(\mathrm{p}=.162)\end{array}$ & $\begin{array}{c}1.640 \\
(p=.185)\end{array}$ & $\begin{array}{c}1.970 \\
(\mathrm{p}=.123)\end{array}$ & 103 \\
\hline \multirow[t]{15}{*}{1978} & \multirow[t]{5}{*}{1122} & $\mathrm{NE}$ & 6.58 & 34.76 & 63.54 & 80 \\
\hline & & NW & 6.45 & 38.75 & 72.58 & 64 \\
\hline & & SW & 6.33 & 37.21 & 71.29 & 90 \\
\hline & & SE & 6.48 & 35.80 & 65.41 & 124 \\
\hline & & $\mathrm{F}=$ & $\begin{array}{c}0.766 \\
(\mathrm{p}=.514)\end{array}$ & $\begin{array}{c}1.475 \\
(\mathrm{p}=.221)\end{array}$ & $\begin{array}{r}3.016 \\
(\mathrm{p}=.030)\end{array}$ & 358 \\
\hline & \multirow[t]{5}{*}{1138} & $\mathrm{NE}$ & 6.70 & 30.87 & 61.81 & 53 \\
\hline & & NW & 6.74 & 29.23 & 63.36 & 35 \\
\hline & & SW & 6.68 & 32.97 & 64.98 & 38 \\
\hline & & SE & 6.61 & 33.89 & 69.69 & 54 \\
\hline & & $\mathrm{F}=$ & $\begin{array}{c}0.140 \\
(p=.936)\end{array}$ & $\begin{array}{c}1.486 \\
(p=.220)\end{array}$ & $\begin{array}{c}1.422 \\
(\mathrm{p}=.238)\end{array}$ & 180 \\
\hline & \multirow[t]{5}{*}{1268} & $\mathrm{NE}$ & 6.74 & 32.57 & 56.03 & 42 \\
\hline & & NW & 6.71 & 35.71 & 64.18 & 31 \\
\hline & & SW & 6.71 & 32.29 & 56.94 & 41 \\
\hline & & SE & 6.41 & 36.00 & 63.76 & 51 \\
\hline & & $\mathrm{F}=$ & $\begin{array}{c}0.852 \\
(p=.467)\end{array}$ & $\begin{array}{c}1.052 \\
(p=.371)\end{array}$ & $\begin{array}{c}1.911 \\
(p=.140)\end{array}$ & 165 \\
\hline
\end{tabular}


TABle I.5. - Continued

\begin{tabular}{|c|c|c|c|c|c|c|}
\hline \multirow[b]{2}{*}{ Year } & \multirow[b]{2}{*}{ Elevation } & \multirow[b]{2}{*}{ Quad } & \multicolumn{4}{|c|}{$\begin{array}{c}\text { Old leaf gall } \\
\text { Quad-means, associated F tests }\end{array}$} \\
\hline & & & Size & Height & Rel. ht. & Number \\
\hline \multirow{15}{*}{1977} & $1122 \mathrm{~m}$ & $\mathrm{NE}$ & $5.45 \mathrm{~mm}$ & $37.95 \mathrm{~cm}$ & $64.63 \%$ & 42 \\
\hline & & NW & 5.43 & 32.33 & 60.76 & 30 \\
\hline & & SW & 5.52 & 31.39 & 57.86 & 54 \\
\hline & & SE & 5.87 & 34.19 & 60.36 & 54 \\
\hline & & $\mathrm{F}=$ & $\begin{array}{c}0.823 \\
(\mathrm{p}=.483)\end{array}$ & $\begin{array}{c}3.082 \\
(p=.029)\end{array}$ & $\begin{array}{c}1.185 \\
(\mathrm{p}=.317)\end{array}$ & 180 \\
\hline & 1138 & $\mathrm{NE}$ & 7.21 & 31.75 & 63.62 & 58 \\
\hline & & NW & 5.77 & 29.69 & 62.79 & 53 \\
\hline & & SW & 6.63 & 30.18 & 62.10 & 52 \\
\hline & & $\mathrm{SE}$ & 7.30 & 28.04 & 59.53 & 61 \\
\hline & & $\mathrm{F}=$ & $\begin{array}{c}3.961 \\
(\mathrm{p}=.009)\end{array}$ & $\begin{array}{c}1.509 \\
(\mathrm{p}=.213)\end{array}$ & $\begin{array}{c}0.689 \\
(p=.560)\end{array}$ & $\overline{224}$ \\
\hline & 1268 & $\mathrm{NE}$ & 6.01 & 35.89 & 65.87 & 126 \\
\hline & & NW & 6.00 & 32.66 & 60.01 & 64 \\
\hline & & SW & 6.11 & 35.36 & 62.31 & 91 \\
\hline & & SE & 5.66 & 33.88 & 59.55 & 118 \\
\hline & & $\mathrm{F}=$ & $\begin{array}{c}1.406 \\
(\mathrm{p}=.241)\end{array}$ & $\begin{array}{c}1.072 \\
(p=.361)\end{array}$ & $\begin{array}{c}2.289 \\
(p=.078)\end{array}$ & $\overline{399}$ \\
\hline \multirow[t]{15}{*}{1978} & 1122 & $\mathrm{NE}$ & 5.25 & 31.46 & 58.82 & 246 \\
\hline & & NW & 5.29 & 32.40 & 61.47 & 241 \\
\hline & & SW & 5.22 & 32.82 & 63.26 & 222 \\
\hline & & $\mathrm{SE}$ & 5.21 & 32.80 & 62.61 & 220 \\
\hline & & $\mathrm{F}=$ & $\begin{array}{c}0.182 \\
(p=.909)\end{array}$ & $\begin{array}{c}0.849 \\
(p=.468)\end{array}$ & $\begin{array}{c}3.962 \\
(\mathrm{p}=.008)\end{array}$ & $\overline{929}$ \\
\hline & 1138 & $\mathrm{NE}$ & 5.00 & 27.58 & 57.76 & 118 \\
\hline & & NW & 5.33 & 28.76 & 61.05 & 76 \\
\hline & & SW & 5.13 & 30.04 & 62.05 & 117 \\
\hline & & SE & 5.04 & 26.23 & 56.52 & 115 \\
\hline & & $\mathrm{F}=$ & $\begin{array}{c}1.438 \\
(p=.231)\end{array}$ & $\begin{array}{c}2.415 \\
(\mathrm{p}=.066)\end{array}$ & $\begin{array}{c}1.744 \\
(\mathrm{p}=.157)\end{array}$ & $\overline{426}$ \\
\hline & 1268 & $\mathrm{NE}$ & 5.14 & 27.59 & 51.05 & 70 \\
\hline & & NW & 5.14 & 31.08 & 56.62 & 63 \\
\hline & & SW & 5.28 & 33.75 & 59.79 & 69 \\
\hline & & $\mathrm{SE}$ & 5.12 & 31.11 & 55.09 & 90 \\
\hline & & $\mathrm{F}=$ & $\begin{array}{c}0.236 \\
(p=.872)\end{array}$ & $\begin{array}{c}3.199 \\
(p=.024)\end{array}$ & $\begin{aligned} 2.510 \\
(p=.059)\end{aligned}$ & $\overline{292}$ \\
\hline
\end{tabular}


TABLE I.5. - CONTINUED

\begin{tabular}{|c|c|c|c|c|c|c|}
\hline \multirow[b]{2}{*}{ Year } & \multirow[b]{2}{*}{ Elevation } & \multirow[b]{2}{*}{ Quad } & \multicolumn{4}{|c|}{$\begin{array}{c}\text { New stem gall } \\
\text { Quad-means, associated F tests }\end{array}$} \\
\hline & & & Size & Height & Rel. ht. & Number \\
\hline \multirow[t]{15}{*}{$1978^{*}$} & \multirow[t]{5}{*}{$1122 \mathrm{~m}$} & $\mathrm{NE}$ & $8.08 \mathrm{~mm}$ & $34.95 \mathrm{~cm}$ & $65.33 \%$ & 112 \\
\hline & & NW & 7.30 & 36.41 & 70.72 & 125 \\
\hline & & SW & 7.92 & 32.67 & 62.52 & 121 \\
\hline & & SE & 9.15 & 35.81 & 68.05 & 153 \\
\hline & & $\mathrm{F}=$ & $\begin{array}{c}5.613 \\
(p=0)\end{array}$ & $\begin{array}{c}2.459 \\
(p=.062)\end{array}$ & $\begin{array}{c}5.259 \\
(p=.001)\end{array}$ & $\overline{511}$ \\
\hline & \multirow[t]{5}{*}{1138} & $\mathrm{NE}$ & 9.30 & 20.50 & 47.60 & 10 \\
\hline & & NW & 6.78 & 27.33 & 55.46 & 9 \\
\hline & & SW & 6.36 & 22.00 & 49.48 & 11 \\
\hline & & SE & 8.14 & 32.14 & 67.95 & 7 \\
\hline & & $\mathrm{F}=$ & $\begin{array}{c}3.772 \\
(p=.020)\end{array}$ & $\begin{array}{c}1.621 \\
(p=.203)\end{array}$ & $\begin{array}{c}1.058 \\
(\mathrm{p}=.380)\end{array}$ & 37 \\
\hline & \multirow[t]{5}{*}{1268} & $\mathrm{NE}$ & 7.27 & 29.36 & 53.03 & 33 \\
\hline & & NW & 7.79 & 28.14 & 51.11 & 28 \\
\hline & & SW & 7.30 & 27.89 & 51.75 & 37 \\
\hline & & $\mathrm{SE}$ & 7.73 & 36.37 & 63.92 & 30 \\
\hline & & $\mathrm{F}=$ & $\begin{array}{c}0.341 \\
(p=.795)\end{array}$ & $\begin{array}{c}2.459 \\
(p=.066)\end{array}$ & $\begin{array}{c}2.197 \\
(\mathrm{p}=.092)\end{array}$ & 128 \\
\hline \multicolumn{7}{|c|}{ Web-rollers } \\
\hline \multirow[t]{15}{*}{$1977+$} & \multirow[t]{5}{*}{1122} & $\mathrm{NE}$ & 11.40 & 39.62 & 73.46 & 5 \\
\hline & & NW & 11.15 & 43.38 & 84.51 & 13 \\
\hline & & SW & 15.25 & 38.10 & 71.39 & 4 \\
\hline & & SE & 11.57 & 33.20 & 66.72 & 7 \\
\hline & & $\mathrm{F}=$ & $\begin{array}{c}1.699 \\
(p=.193)\end{array}$ & $\begin{array}{c}1.626 \\
(p=.208)\end{array}$ & $\begin{array}{c}2.201 \\
(p=.113)\end{array}$ & 29 \\
\hline & \multirow[t]{5}{*}{1136} & $\mathrm{NE}$ & 13.71 & 35.81 & 76.04 & 24 \\
\hline & & NW & 11.82 & 39.65 & 78.31 & 38 \\
\hline & & SW & 11.83 & 33.99 & 66.28 & 24 \\
\hline & & SE & 11.95 & 34.47 & 66.53 & 22 \\
\hline & & $\mathrm{F}=$ & $\begin{array}{c}1.017 \\
(\mathrm{p}=.389)\end{array}$ & $\begin{array}{c}2.514 \\
(\mathrm{p}=.0625)\end{array}$ & $\begin{array}{c}4.456 \\
(p=.005)\end{array}$ & $\overline{108}$ \\
\hline & \multirow[t]{5}{*}{1268} & $\mathrm{NE}$ & 14.50 & 36.83 & 65.91 & 1 \\
\hline & & NW & 11.33 & 45.92 & 82.86 & 6 \\
\hline & & SW & 7.00 & 33.02 & 61.90 & 1 \\
\hline & & SE & - & - & - & 0 \\
\hline & & $\mathrm{F}=$ & $\begin{array}{c}1.311 \\
(p=.337)\end{array}$ & $\begin{array}{c}3.012 \\
(p=.124)\end{array}$ & $\begin{array}{c}2.846 \\
(p=.135)\end{array}$ & 9 \\
\hline
\end{tabular}

*New stem galls not measured in 1977.

tWeb-rollers not measured in 1978. 
TABLE I.5. - CONTINUED

\begin{tabular}{|c|c|c|c|c|c|c|}
\hline \multirow[b]{2}{*}{ Year } & \multirow[b]{2}{*}{ Elevation } & \multirow[b]{2}{*}{ Quad } & \multicolumn{4}{|c|}{$\begin{array}{c}\text { Old stem gall } \\
\text { Quad-means, associated F tests }\end{array}$} \\
\hline & & & Size & Height & Rel. ht. & Number \\
\hline \multirow[t]{15}{*}{$1977 \ddagger$} & \multirow[t]{5}{*}{$1122 \mathrm{~m}$} & $\mathrm{NE}$ & $17.00 \mathrm{~mm}$ & $22.86 \mathrm{~cm}$ & $42.86 \%$ & 1 \\
\hline & & NW & 14.67 & 34.72 & 67.66 & 3 \\
\hline & & SW & - & - & - & 0 \\
\hline & & SE & 12.25 & 20.96 & 38.66 & 4 \\
\hline & & $\mathrm{F}=$ & $\begin{array}{c}0.996 \\
(p=.432)\end{array}$ & $\begin{array}{c}3.505 \\
(\mathrm{p}=.112)\end{array}$ & $\begin{array}{c}14.490 \\
(p=.008)\end{array}$ & 8 \\
\hline & \multirow[t]{5}{*}{1138} & $\mathrm{NE}$ & 14.00 & 25.40 & 57.57 & 2 \\
\hline & & NW & 14.25 & 30.78 & 64.42 & 4 \\
\hline & & SW & 15.50 & 22.86 & 47.70 & 6 \\
\hline & & SE & 17.40 & 16.76 & 42.97 & 5 \\
\hline & & $\mathrm{F}=$ & $\begin{array}{c}0.370 \\
(p=.776)\end{array}$ & $\begin{array}{c}1.395 \\
(p=.289)\end{array}$ & $\begin{array}{c}0.800 \\
(p=.516)\end{array}$ & 17 \\
\hline & \multirow[t]{5}{*}{1268} & $\mathrm{NE}$ & 13.18 & 35.92 & 64.48 & 11 \\
\hline & & NW & 12.12 & 33.50 & 63.34 & 8 \\
\hline & & SW & 14.55 & 34.87 & 62.23 & 8 \\
\hline & & SE & 12.50 & 39.07 & 67.67 & 11 \\
\hline & & $\mathrm{F}=$ & $\begin{array}{c}0.842 \\
(\mathrm{p}=.480)\end{array}$ & $\begin{array}{c}0.430 \\
(p=.733)\end{array}$ & $\begin{array}{c}0.120 \\
(p=.948)\end{array}$ & 38 \\
\hline \multirow[t]{15}{*}{1978} & \multirow[t]{5}{*}{1122} & $\mathrm{NE}$ & 10.86 & 28.20 & 53.29 & 65 \\
\hline & & NW & 10.85 & 28.21 & 56.02 & 66 \\
\hline & & SW & 11.30 & 30.68 & 57.61 & 50 \\
\hline & & SE & 11.24 & 32.87 & 63.42 & $\underline{110}$ \\
\hline & & $\mathrm{F}=$ & $\begin{array}{c}0.907 \\
(p=.438)\end{array}$ & $\begin{array}{c}2.977 \\
(p=.032)\end{array}$ & $\begin{array}{c}3.837 \\
(\mathrm{p}=.010)\end{array}$ & 291 \\
\hline & \multirow[t]{5}{*}{1138} & $\mathrm{NE}$ & 11.74 & 30.30 & 58.47 & 27 \\
\hline & & NW & 12.88 & 30.18 & 58.24 & 17 \\
\hline & & SW & 12.50 & 30.00 & 56.10 & 18 \\
\hline & & SE & 11.67 & 28.50 & 57.14 & 18 \\
\hline & & $\mathrm{F}=$ & $\begin{array}{c}0.432 \\
(p=.731)\end{array}$ & $\begin{array}{c}0.085 \\
(p=.968)\end{array}$ & $\begin{array}{c}0.042 \\
(p=.989)\end{array}$ & 80 \\
\hline & \multirow[t]{5}{*}{1268} & $\mathrm{NE}$ & 11.80 & 35.07 & 59.53 & 30 \\
\hline & & NW & 11.21 & 27.76 & 47.94 & 34 \\
\hline & & SW & 11.42 & 33.96 & 57.64 & 45 \\
\hline & & SE & 11.86 & 35.41 & 58.87 & 56 \\
\hline & & $\mathrm{F}=$ & $\begin{array}{c}0.503 \\
(p=.681)\end{array}$ & $\begin{array}{c}2.349 \\
(\mathrm{p}=.075)\end{array}$ & $\begin{array}{c}2.187 \\
(\mathrm{p}=.092)\end{array}$ & 165 \\
\hline
\end{tabular}

‡Excluding bark-covered old stem galls in 1977.

The largest new leaf galls occurred in the SW quad at all elevations in 1977, although not with statistical significance except at $1122 \mathrm{~m}$. Old leaf galls were also larger in the NW and SW in 1978. This similarity to 1977 patterns was expected, since old leaf galls are largely composed of previous-year new galls. In general, the NE or SE quad held the largest galls, except for old leaf galls at $1268 \mathrm{~m}$ in 1977 (where SW galls were large and SE galls were small).

Among leaf galls in 1978, the greater the quad density, the smaller the gall (Table I.5). The correlation between number of galls per quad and quad-mean gall size was negative at all elevations for both new and old leaf galls, ranging from -.35 to -.93 . Old leaf-gall 
height was significantly different among quads at $1122 \mathrm{~m}$, with west-facing galls closer to the ground. Differences at other elevations were not significant (with west-facing galls intermediate in height between the SE and NE quads at $1138 \mathrm{~m}$, and SE-NW galls closest to the ground at $1268 \mathrm{~m}$ ). Correlations between elevations were all positive for relative height (from $r(2)=.47$ to $r(2)=.70$ ), indicating that relative height patterns were consistent among elevations.

The above observations revealed no consistent leaf-gall size disparities among quads, contrary to residence in a particular quad conferring predictable gall characteristics. Instead, relationships among quad-mean variables were examined. Results showed that trends were similar between 1977 and 1978 for leaf gall density versus absolute height: denser galls were further from the ground $(\mathrm{r}(10)=.833$ and .552 , respectively). Correlations involving size were disparate, however, between 1977 and 1978. In 1977, larger galls tended to be denser $(r(10)=.409)$ and higher $(r(10)=.556)$, whereas in 1978 they were sparser $(r(10)=-.713)$ and lower $(r(10)=-.417)$.

The above discrepancy was related to differences in mean leaf gall size each year: 4.77-6.29 mm quad-mean diameter in 1977 versus 6.33-6.74 $\mathrm{mm}$ in 1978 . At low densities $(<3$ new leaf galls per quad per plant) the correlation between density and gall diameter was $r(9)=+.495$, whereas at high densities it was $r(11)=-.599$ (Fig. I.3). Thus, size decreased as density increased in dense quads whereas sparse quad densities bore no significant relation to gall size.

The relation between leaf gall height above the ground and gall diameter was similarly clarified by stratifying with respect to gall diameter (Fig. I.4). For galls measuring greater than $6.3 \mathrm{~mm}, \mathrm{r}(10)=-.806$; for galls $5.4-6.3 \mathrm{~mm}, \mathrm{r}(6)=-.705$; and for galls less than $5.4 \mathrm{~mm}, \mathrm{r}(2)=-.413$. Thus, gall size decreased as height above the ground increased, with the tightest coupling at the largest gall sizes. Among all quads with a given leaf gall density, galls higher with respect to the plant were larger $(r(21)=-.503)$, at variance with findings not holding constant gall density. Similarly, the greater the number of galls per quad, the smaller the gall size when examined at constant gall heights $(r(21)=-.446)$.

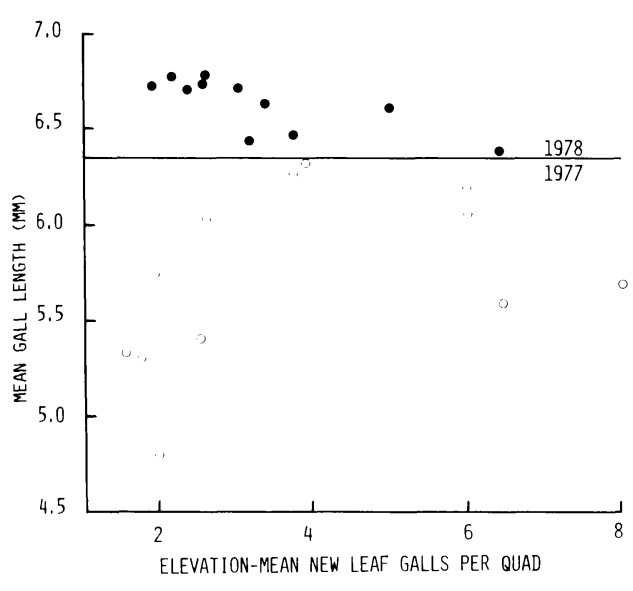

Fig. I.3. The relationship between elevationmean number of new leaf galls per quad and mean gall length, for $T$. stenolepis in the New York Mountains, April 1977 and April 1978.

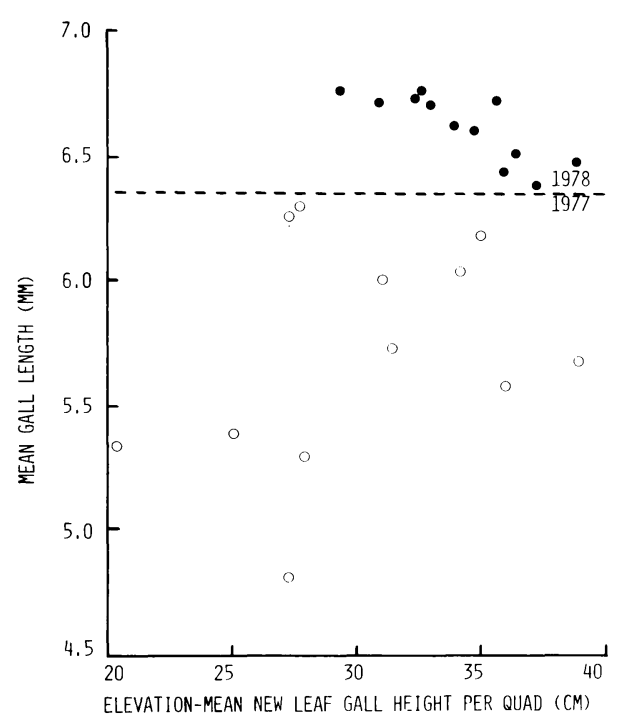

Fig. I.4. The relationship between elevationmean new leaf gall height per quad and mean gall length, for T. stenolepis in the New York Mountains, April 1977 and April 1978. 
This minimized the possibility of dense leaf galls being consistently higher, and thus smaller.

Analogous correlations between stem gall height or relative height and gall size, holding constant stem gall density, were inconsistent. New stem galls showed no correlation $(\mathrm{r}(9)=.020$ ), fuzzy (year-old) stem galls in 1977 showed a negative (nonsignificant) correlation $(\mathrm{r}(9)=-.321)$, and old stem galls in 1978 showed significant positive correlation $(\mathrm{r}(9)=.642)$.

The correlation between stem gall density and gall length, holding constant gall height, was consistently negative for old stem galls $(\mathrm{r}=-.625, \mathrm{p}=.001$ pooled over 1977 and 1978 ) but insignificantly positive for new stem galls. Thus old dense stem galls were smaller than old sparse stem galls. The negative correlation was even stronger in 1978 $(\mathrm{r}(9)=-.711)$. The persistence of this relation implied its relevance between years as well as among quads and plants.

Gall size and shape depended not only on the above factors of insect identity and behavior, but seasonality. Time lags in gall emergence produced gall flushes, whose characteristics varied according to synchrony between emergence and plant growth. Inspection of stem gall length histograms for each plant sampled April 1978 at $1122 \mathrm{~m}$ in the New York Mountains showed three distinct modes. Sometimes all three were shifted downward so that first flush gall size appeared to equal that of second flush galls on plants with generally greater growth. Thus, plant-mean stem gall sizes were uninformative as they failed to distinguish between, for example, a plant with all three modes, each consisting of shorter galls, and a plant with only third-flush galls.

Annual weather differences were another seasonal factor in gall size variation. For example, 1978 new leaf galls were significantly larger than in 1977 regardless of elevation or whether sampled in April or May of 1978 (all sampling was in April in 1977). Winter rainfall in 1978 significantly exceeded that in 1977 , indicated by a paired comparison t-test $(\mathrm{t}=10.95, \mathrm{p}<.001)$. Larger galls are expected in rainy years since overall greater plant growth is transmitted to the galls.

Slope and elevation also affected plant growth and hence gall development. Statistically controlling for species composition among sites suppressed potential spurious effects on gall size. Adjusted deviations showed that steep slopes and intermediate elevations were associated with the longest galls and the widest chambers in both northern and southern localities (Table I.6). Deviations due to species of Tetradymia were 1.3-5 times larger in the north than in the south (mean ratio $=2.89 \pm 0.790$ ). Deviations due to slope or elevation were 2.4-8.2 times larger in the south than in the north (mean ratio $=4.60 \pm$ $1.315)$. Thus, the plant effect predominated in the north while the geographic effect predominated in the south.

\section{DISCUSSION}

Significant differences in Tetradymia gall morphology were associated with locality, month and year of collection, and height on the plant. A multi-way analysis of variance showed that after host plant species was held constant, stem gall inhabitant influenced gall appearance next most strongly, followed by degree of slope (a moisture effect), and elevation (a temperature effect). Latitude per se was unimportant.

Early instar G. tetradymiella larvae were found in shortened and/or narrowed stem galls at all sites except Beekley Road. Washburn and Cornell (1981) also found that the earlier a cynipid oak gallmaker was attacked by a parasitoid, the smaller the mature gall. This suggested a positive association between feeding and gall size. Seemingly contradictory 
evidence, such as when the length of late instar galls exceeded that of pupal galls (Table I.4), was reconciled by taking into account plant growth spurts. Galls initiated appreciably before or after a rapid elongation period (signalled by advanced or retarded gall insect development relative to the population mean) were likely to be stunted.

To test the feeding/gall size connection I indexed feeding duration by ranking relative body sizes of the gall inhabitants from one, for early instars, to four, for Copidosoma n. sp. near deceptor Miller (polyembryonic encyrtid parasitoids which grossly enlarge the host's body). Empty galls were ranked as two (the mean value of 1,2 , and 3 ) to encompass mortality during a variety of stages. Gall lengths (adjusted with respect to Tetradymia species, slope, elevation, and latitude using covariance analysis) were then averaged across all five sites and their correlation with feeding duration ranks calculated. The positive coefficient, $r(4)=.804$, suggested that the longer a gall is fed upon, the greater its growth.

Alterations in feeding duration and/or intensity depend on type of parasitization. Hunter and Stoner (1975) found that Trichoplusia ni (Hübner) parasitized by Copidosoma truncatellum (Dalman) fed two days longer than control larvae and hence consumed more total food, yet the final parasitized $T$. ni weight did not differ from mean control larva weight. Beegle (1971), on the other hand, found that T. ni parasitized by Hyposoter exiguae (Viereck) did not consume more total food, and the final parasitized weight was significantly less than that of control larvae. If a parasitized larva is to maintain normal body weight (including the weight of its endoparasitoids), it must feed more to compensate for the extra link in the food chain introduced by the parasitoid. The relatively large changes in stem gall size induced by small changes in pest feeding suggest the use of galls as a "biological amplifier" of parasitoid effects in biological control studies.

TABLE I.6. MULTIPLE CLASSIFICATION ANALYSIS OF STEM GALL LENGTH AND CHAMBER WIDTH BY HOST PLANT SPECIES, SLOPE, AND ELEVATION OF STUDY SITE, WITHIN EACH LATITUDE CLASSIFICATION, SEPTEMBER 1980

\begin{tabular}{|c|c|c|c|c|c|c|c|c|}
\hline \multirow[b]{4}{*}{ Class } & \multicolumn{8}{|c|}{ Deviation from grand mean $(\mathrm{mm})$} \\
\hline & \multicolumn{4}{|c|}{ Gall length } & \multicolumn{4}{|c|}{ Chamber width } \\
\hline & \multicolumn{2}{|c|}{ North } & \multicolumn{2}{|c|}{ South } & \multicolumn{2}{|c|}{ North } & \multicolumn{2}{|c|}{ South } \\
\hline & Raw & Adj. & Raw & Adj. & Raw & Adj. & Raw & Adj. \\
\hline \multicolumn{9}{|l|}{ Tetradymia } \\
\hline $\begin{array}{l}\text { stenolepis } \\
\text { axillaris }\end{array}$ & $\begin{array}{r}1.03 \\
-1.76\end{array}$ & $\begin{array}{r}0.86 \\
0.89 \\
-1.46 \\
-1.53\end{array}$ & $\begin{array}{r}-0.17 \\
0.74\end{array}$ & $\begin{array}{r}0.18 \\
-0.76\end{array}$ & $\begin{array}{r}0.59 \\
-1.01\end{array}$ & $\begin{array}{r}0.50 \\
0.52 \\
-0.85 \\
-0.90\end{array}$ & $\begin{array}{r}0.22 \\
-0.95\end{array}$ & $\begin{array}{r}0.15 \\
-0.67\end{array}$ \\
\hline $\begin{array}{l}\text { Slope† } \\
\text { level } \\
\text { steep }\end{array}$ & $\begin{array}{r}-0.76 \\
1.64\end{array}$ & $\begin{array}{r}-0.24 \\
0.52\end{array}$ & * & * & $\begin{array}{r}-0.32 \\
0.69\end{array}$ & $\begin{array}{r}-0.04 \\
0.09\end{array}$ & $\begin{array}{r}-0.32 \\
0.35\end{array}$ & $\begin{array}{r}-0.20 \\
0.22\end{array}$ \\
\hline $\begin{array}{l}\text { Elevation } \ddagger \\
\text { low } \\
\text { intermediate } \\
\text { high }\end{array}$ & $\begin{array}{r}-0.22 \\
1.64 \\
-0.84\end{array}$ & $\begin{array}{r}-0.51 \\
0.51 \\
-0.19\end{array}$ & $\begin{array}{r}* \\
1.35 \\
-1.49\end{array}$ & $\begin{array}{r}* \\
1.40 \\
-1.55\end{array}$ & $\begin{array}{r}0.31 \\
0.69 \\
-0.42\end{array}$ & $\begin{array}{c}-0.24 \\
0.07 \\
0\end{array}$ & * & $*$ \\
\hline Grand mean: & & 40 & & 79 & & 32 & & 63 \\
\hline
\end{tabular}

*Slope/elevation effect in south heuristically assigned to slope for gall length and to elevation for chamber width on basis of similar trends in north.

†Level = less than $1 \mathrm{~m}$ per $30 \mathrm{~m}$; steep = more than $1 \mathrm{~m}$ per $30 \mathrm{~m}$.

$\ddagger$ Low $=800-999 \mathrm{~m} ;$ intermediate $=1000-1199 \mathrm{~m} ;$ high $=1200-1399 \mathrm{~m}$. 
Differential growth among plant parts also influenced gall form. For example, Scrobipalpa leaf galls from densely-populated plant quads at the New York Mountains in 1978 were smaller than their better-spaced cohorts. If increased infestation signaled stress on the plant, leaves and hence galls produced would be smaller in sectors of greatest stress. The impact of gall infestation on Tetradymia, however, remains in question (cf. Hartman, 1983b). Plants with the smallest galls (indicating less growth) were most heavily infested, suggesting already-stressed plants were more vulnerable to further stress. The gall size decrease with gall density only in dense quads was analagous to findings by Raske and Bryant (1976): correlations between birch casebearer density and bud density were significant only at the highest population levels, attributed to reduced food and oviposition site availability.

A decrease in leaf gall size as height above the ground increased implied height-limited gall growth, through either increased insolation and/or reduced access to soil moisture. Zimmermann (1978) discovered that hydraulic constrictions at the point where a branch joins a tree trunk reduced rate of water flow. Mean moisture of $T$. stenolepis lower leaves was 66.5 percent of their wet weight while that of upper leaves was 57.5 percent $(t=3.602$, $\mathrm{p}=.0407)$. The relation between quad-mean leaf gall size and height became positive only at constant gall density. Leaf gall density and size correlations at constant gall height, however, did not change sign from the uncontrolled case. The smallness of dense leaf galls, therefore, seemed biologically significant and may further the "galls as stress" argument.

The lack of a consistent relation between number of G. tetradymiella stem galls in a given quad and their quad-mean size suggested that gall density did not influence gall length. Stem gall size may be influenced more by the location of the basal stem portion than by the location of the gall itself. Depending on the plant, branches originate in the same quad as their galls, a different quad, or all from the basal trunk region.

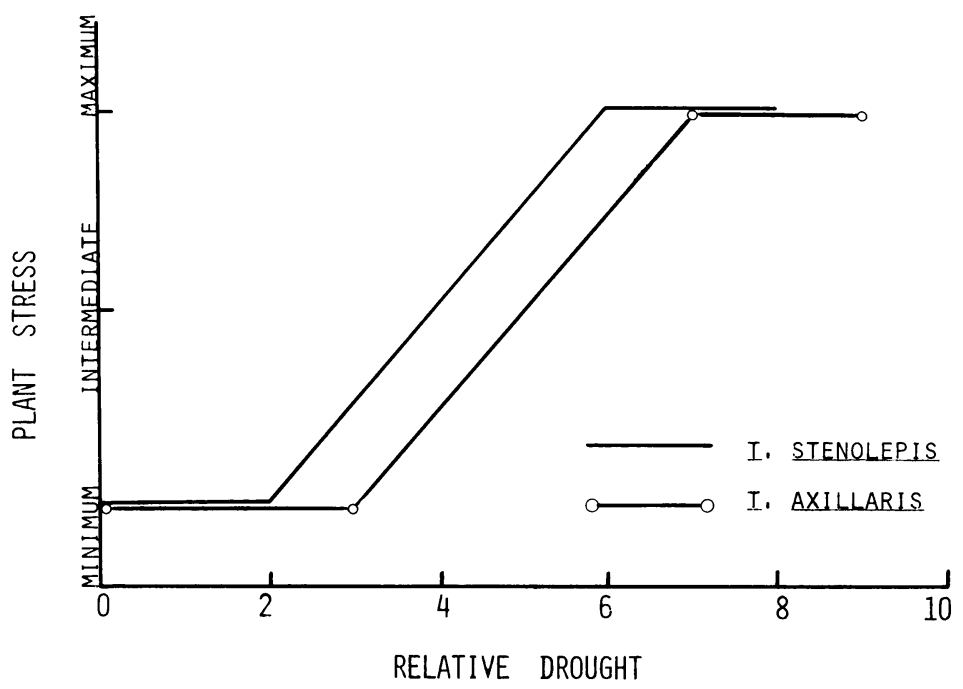

Fig. I.5. Theoretical effect of relative drought on Tetradymia species with differing degrees of xeric adaptation. After Boyko (1955). 
New stem-gall size and density remained unrelated when gall height was held constant, perhaps due to the aforementioned emergence of stem galls in "flushes." The emergence of many plant galls coincides with periods of greatest plant growth. In the summer when most growth slows, energy that would normally sustain pre-existent plant organs may be redirected to the development of a second flush of galls (Russo, 1979). Washburn and Cornell (1981) also observed a second flush of oak sucker shoots during the critical attack period of cynipid gallmakers.

Waldbauer (1978) posited that the modes of a polymodal emergence curve might represent groups of individuals with different diapause intensities. Many insects with such curves are associated with marginal areas where conditions tend to vary unpredictably. Partitioning progeny between early and late emergers might allow at least some to escape unexpected detrimental conditions. Members of G. tetradymiella conformed to "Type A" polymodality, where all individuals which enter diapause in the same year terminate it the following growth season, producing a bimodal or trimodal emergence curve.

Topographic variables affected gall size and shape, but not as strongly as species of plant producing the gall or species of insect occupying the gall. Slope and elevation effects remained significant after holding constant plant and insect species, especially in the south. Southern study sites approached Tetradymia range limits most closely and were probably more stressful to the plant. In terms of Figure I.5 (after Boyko, 1955), southern localities may occupy a position along the drought axis at ca. seven. Here both species would experience similarly high levels of stress but be sensitive to minor variations in moisture mediators, such as slope or elevation, which move their positions leftward. Northern sites, on the other hand, might fall midrange on the drought axis, thus leading to larger species differences and less sensitivity to local topographic features.

\section{LITERATURE CITED}

BEEGLE, C. C.

1971. Effect of a nuclear polyhedrosis virus on the relationship between Trichoplusia ni and the parasite, BOYKO, $\mathrm{H}$. Hyposoter exiguae. Ph.D. thesis. Univ. of California, Riverside.

1955. Climatic, ecoclimatic and hydrological influences on vegetation. In: Plant Ecology. Proc. Montpellier Symp., Paris; UNESCO. p. 43.

COSENS, A.

1913. Insect galls. Can. Entomol. 45:380-384.

HARTMAN, $\mathrm{H}$.

1982a. Quantitative ecology of gall-forming Lepidoptera on the Mojave Horsebrush, Tetradymia, Vol. I. Ph.D. thesis. Univ. of California, Riverside.

1982b. Quantitative ecology of gall-forming Lepidoptera on the Mojave Horsebrush, Tetradymia, Vol. II. Data listings. Univ. of California, Riverside.

1983b. Ecology of gall-forming Lepidoptera on Tetradymia II. Plant stress effects on infestation intensity. Hilgardia (this issue).

1983c. Ecology of gall-forming Lepidoptera on Tetradymia III. Within-plant horizontal and vertical distribution. Hilgardia (this issue).

HUNTER, K. W., Jr., and A. STONER.

1975. Copidosoma truncatellum: Effect of parasitization on food consumption of larval Trichoplusia ni. Environ. Entomol. 4:381-382.

MANI, M. S.

1964. Ecology of plant galls. The Hague, Dr. W. Junk. Netherlands:434 p. 
INIE, N. H., C. H. HULL, J. G. JENKINS, K. STEINBRENNER, and D. H. BENT.

1975. SPSS: Statistical Package for the Social Sciences. New York: McGraw-Hill. 675 p.

RASKE, A. G., and D. G. BRYANT.

1976. Distribution of overwintering birch casebearer larvae, Coleophora fuscedinella, on white birch (Lepidoptera: Coleophoridae). Can. Entomol. 108:407-414.

ROBERT, P. C., and P. BLAISINGER.

1978. Role of non-host plant chemicals in the reproduction of an oligophagous insect: the sugar beet moth Scrobipalpa ocellatella (Lepidoptera: Gelechiidae). Entomol. Exp. Appl. 24:432-436.

RUSSO, R. A.

1979. Plant galls of the California region. Pacific Grove, California: Boxwood Press. 203 p.

STROTHER, J. L.

1974. Taxonomy of Tetradymia (Compositae: Senecioneae). Brittonia 26:177-202.

WALDBAUER, G. P.

1978. Phenological adaptation and the polymodal emergence patterns of insects. In: Evolution of insect migration and diapause (H. Dingle, ed.). New York: Springer-Verlag. 284 p.

WASHBURN, J. O., and H. V. CORNELL.

1981. Parasitoids, patches, and phenology: their possible role in the local extinction of a cynipid gall wasp population. Ecology 62:1595-1607.

ZIMMERMANN, M. H.

1978. Structural requirements for optimal water conduction in tree stems. In: Tropical trees as living systems (P. B. Tomlinson and M. H. Zimmermann, eds.). New York: Cambridge Univ. Press. pp. 517-532. 